\title{
Self-Organization and Cooperativity of Weakly Coupled Molecular Motors under Unequal Loading
}

\author{
Jan Brugués ${ }^{1,2}$ and Jaume Casademunt ${ }^{1}$ \\ ${ }^{1}$ Departament d'ECM, Universitat de Barcelona, Avinguda Diagonal 647, E-08028 Barcelona, Spain \\ ${ }^{2}$ Laboratoire Physico-Chimie Théorique, UMR CNRS Gulliver 7083, ESPCI, 10 rue Vauquelin, 75005 Paris, France
}

(Received 2 July 2008; published 17 March 2009)

\begin{abstract}
We study the collective dynamics of Brownian motors moving on a one-dimensional track when an external load is applied to the leading motor. Motors are driven by a two-state ratchet mechanism, which is appropriate to single-headed kinesins, and their relative motion is only constrained by their mutual interaction potential (weak coupling). We show that unequal loading enhances cooperativity, leading to the formation of clusters with velocities and efficiencies higher than those predicted by simple superposition. When a weak attraction between motors is present, we find nonmonotonic collective velocity-force curves, hysteretic phenomena, and a dynamic self-regulation mechanism that selects the cluster size for optimal performance.
\end{abstract}

DOI: 10.1103/PhysRevLett.102.118104

The collective behavior of molecular motors plays a crucial role in different biological processes in intracellular transport [1,2]. Most studies of collective effects of motors have focused on situations in which the cargo to which they are attached is rigid or elastic, corresponding to a strong coupling between motors [3-6], including the case of small numbers of motors $[7,8]$. In other circumstances, the collective action of small groups of motors is required to pull on fluidlike cargoes, such as in the formation of membrane tubes [9-13] or in vesicle transport. In this case, motors are weakly coupled in the sense that they are free to move relative to each other along the fluidlike cargo. In addition, only the few motors that pull with a normal component to the membrane may exert force directly on the cargo. Since motors under load are slower, the unloaded motors will pile up behind forming a cluster. In many cases the cooperation of these additional motors may be required to overcome the total external force [11]. Force transmission from the unloaded motors is then crucial and results from motor-motor interactions. Despite recent theoretical progress [11-13], the precise mechanism of force transmission, and the connection between interactions and collective performance of such motor clusters is poorly understood. A recent approach based on biased random walkers on a lattice has found a strong sensitivity of collective velocity-force curves to motor interactions [12]. However, the relation between stochastic dynamics and physical interactions between motors in these models is unclear. A more mechanistic approach where internal degrees of freedom of motors are resolved and coupled to positional degrees of freedom through explicit interactions could shed new light. We focus on the isothermal ratchet model for a motor [1416], which in turn is a paradigm of nonequilibrium transport. In the biological context it is directly relevant to monomeric kinesin (KIF1A)[17,18], a family of motors that play an essential role in axonal transport. Large ensembles of KIF1A have been recently studied from a
PACS numbers: 87.10.-e, 05.40.-a, 87.16.Nn, 87.17.Aa

statistical physics point of view [19]. Experiments with small clusters of KIF1A have found a remarkable increase of the velocities in the case of rigid coupling [18]. Given the connection between disorders in intracellular traffic and neurodegenerative diseases, understanding of the collective dynamics of such motors is potentially important for biomedical implications.

We consider a set of $N$ motors moving along a onedimensional track. An external force $F$ opposing motion is applied onto the leading motor. For simplicity, and to better isolate the cooperative mechanisms, motors are assumed infinitely processive: they walk on the track without detaching from it. Biological applications will eventually require to relax this assumption. We assume that motors interact with an arbitrary, short-ranged (nonbinding) potential including a hard-core repulsive part. We consider the simplest case for single-motor dynamics within a twostate isothermal ratchet [14-16]. We define Langevin equations for the motor positions

$$
\lambda \dot{x}_{i}=-U_{i}^{\prime}\left(x_{i}, k_{i}\right)-\sum_{k \neq i} W^{\prime}\left(x_{i}-x_{k}\right)-F \delta_{1 i}+\zeta_{i}(t)
$$

where $i=1, \ldots, N, k_{i}$ are discrete stochastic variables that label the two internal states of each motor, characterized by a bound state potential $U_{i}\left(x_{i}, 1\right)$ and a weakly bound state potential $U_{i}\left(x_{i}, 2\right)$. Each motor switches states independently: transition rates of the $i$ th motor depend only on $x_{i} . W(\xi)$ is the interacting potential between motors. The friction coefficient $\lambda$ is assumed to be the same for the two states, so thermal noise is a Gaussian process with $\left\langle\zeta_{i}(t) \zeta_{j}\left(t^{\prime}\right)\right\rangle=2 k_{B} T \lambda \delta_{i j} \delta\left(t-t^{\prime}\right)$, with a noise strength $D=k_{B} T / \lambda$. For simplicity and better understanding of the cooperative mechanisms, we take $U_{i}(x, 1)$ (hereinafter the $U_{1}$ state) as a fully asymmetric sawtooth potential with period $\ell$ and height $U$, and a sliding velocity $v=U /(\lambda \ell)$ (see Fig. 1). The state $U_{i}(x, 2)$, (hereinafter the $U_{2}$ state) is a weakly bound state modeled by a flat potential. 


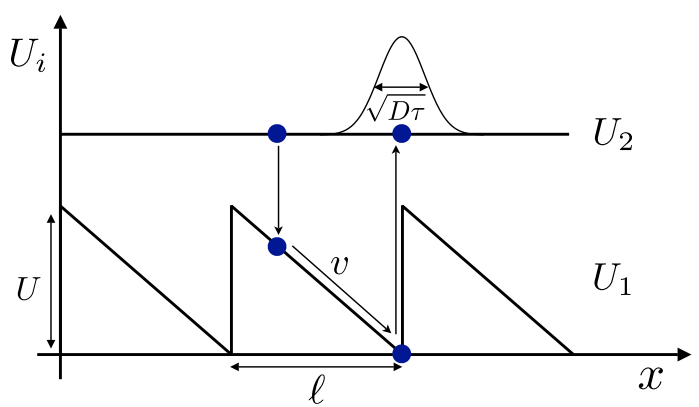

FIG. 1 (color online). Two-state potentials for each motor.

Excitations from $U_{1}$ to $U_{2}$ are localized at the minimum of $U_{1}$ and are assumed instantaneous upon reaching that point (i.e., much faster than any other process in the motor cycle). This corresponds to a far-from-equilibrium condition $\Delta \mu \gg k_{B} T$, where $\Delta \mu$ is the chemical potential difference for ATP hydrolysis (thermal activations are neglected) $[15,16]$. The lifetime of $U_{2}$ is $\tau$ and deexcitations are delocalized. There are two relevant dimensionless parameters: $\alpha \equiv v \tau / \ell$, the ratio of the lifetime of $U_{2}$ to the characteristic sliding time of $U_{1}$, and $\beta \equiv \ell /(4 D \tau)^{1 / 2}$, the ratio of the track period to the diffusive length in $U_{2}$. In the limit of weak noise, the stall force and the velocity at zero load are $F_{s}^{0}=\lambda v \min \left[1, \frac{1}{2 \alpha}\right]$ and $V_{1}^{0}(0)=\frac{v}{1+2 \alpha}$ [20]. Our observables are the cluster velocity, $V_{N}(F) \equiv\left\langle\dot{x}_{1}\right\rangle$, and the cluster efficiency, which in the biological context reads $\eta_{N}(F)=\frac{F V(F)}{r_{N}(F) \Delta \mu}$, where $r_{N}(F)$ is the chemical reaction rate, in our case the number of excitations per unit time.

Mean-field.-We first consider a mean-field (MF) ansatz of the form $W^{\prime}(\xi) \simeq W^{\prime}(\langle\xi\rangle)$, which neglects correlations between positional and internal degrees of freedom of different motors. The steady state solution implies that the force between two motors is constant, and each motor behaves as an isolated motor subject to an equal share $F / N$ of the external force. The problem is then reduced to the single-motor one with $V_{N}(F)=V_{1}(F / N)$ and $F_{N}(V)=$ $N F_{1}(V)$. Similarly, the energy consumption satisfies $r_{N}(F)=N r_{1}(F / N)$, so the collective efficiency is $\eta_{N}(F)=\eta_{1}(F / N)$. The conditions for the validity of MF require a sufficiently smooth and long-ranged interaction and/or a sufficiently strong entropic repulsion $(\beta \lesssim 1)$. Convergence to MF behavior in the appropriate limit has been studied in detail [20].

Hard-core repulsion.-Significant deviations from MF may be expected for short-ranged repulsive interactions [12] and for small or moderate noise strength. In our model we find that correlations typically improve the collective performance with respect to MF: $V_{N}(F)>V_{1}(F / N)$ and $F_{N}(V)>N F_{1}(V)$, a scenario that we refer to as "enhanced cooperativity." While this effect may be quantitatively dramatic, the opposite inequality occurs in very limited parameter ranges, and remains quantitatively small. Figure 2 shows two illustrative examples. The inset shows the predominance of enhanced cooperativity for variable

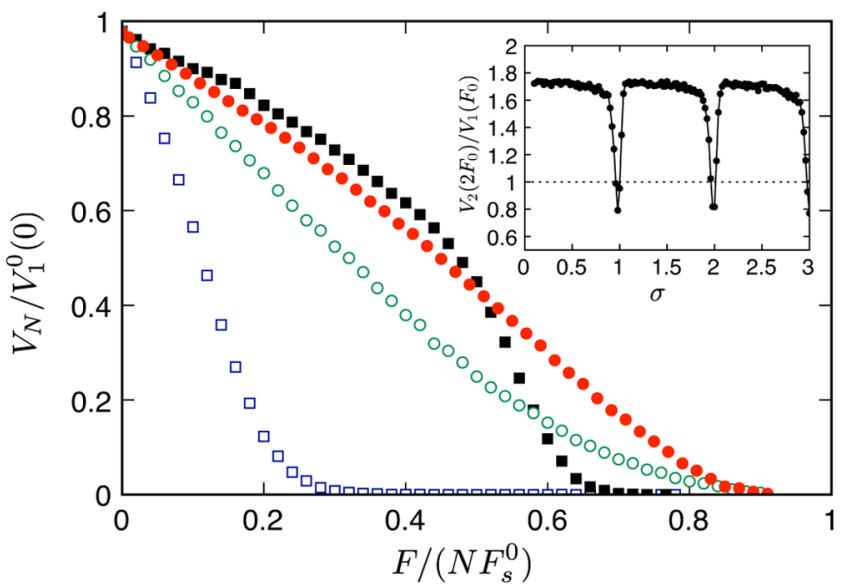

FIG. 2 (color online). Velocity-force relationship for $N=1,2$ for $\alpha=0.15$ and hard-core repulsion (repulsive part of a Lennard-Jones potential $W(\xi)=4 \epsilon\left(\left(\frac{\sigma}{\xi}\right)^{12}-\left(\frac{\sigma}{\xi}\right)^{6}\right)$ truncated at $\left.\xi=2^{1 / 6} \sigma\right)$, with $\sigma=0.2 \ell$ and $\epsilon=1 / 4$. Circles correspond to $\beta=10$; empty circles, $N=1$ and $N=2$ with MF; solid circles, $N=2$. Squares correspond to $\beta=44.7$; empty squares, $N=1$ and $N=2$ with $\mathrm{MF}$; solid squares, $N=2$. Axes are normalized by $D=0$ values of the 1 -motor stall force and free velocity (see text). The inset shows the dependence on the hardcore size $\sigma$ of the gain with respect to MF for $F_{0}=\frac{1}{3} F_{s}^{0}$ and $\beta=14$. Units are such that $v=3, \lambda=1$ and $\ell=1$.

hard-core size $\sigma$. As we increase $N$ we find that $V_{N}(F)$ converges rather quickly to a limiting curve $V_{N} \rightarrow V_{\infty}(f)$, where $f \equiv F / N$, recovering the extensivity of $\mathrm{MF}$, but with $V_{\infty}(f) \neq V_{1}(f)$ (see Fig. 3). This is an important difference with respect to the prediction of the lattice model [12], where $V_{N}(F)$ was found to saturate with $N$ to a limiting curve $V_{N}(F) \rightarrow \tilde{V}(F)$ independent of $N$. For KIF1A, in the experimental conditions of $[17,18]$, the extensivity of the effect of motors is guaranteed, since the noise is very strong, with $\beta \simeq 0.3\left(D=40 \mathrm{~nm}^{2} / \mathrm{s}, \tau=\right.$ $0.5 \mathrm{~ms}$ and $\ell=8 \mathrm{~nm}$ ) and MF holds. In general, which

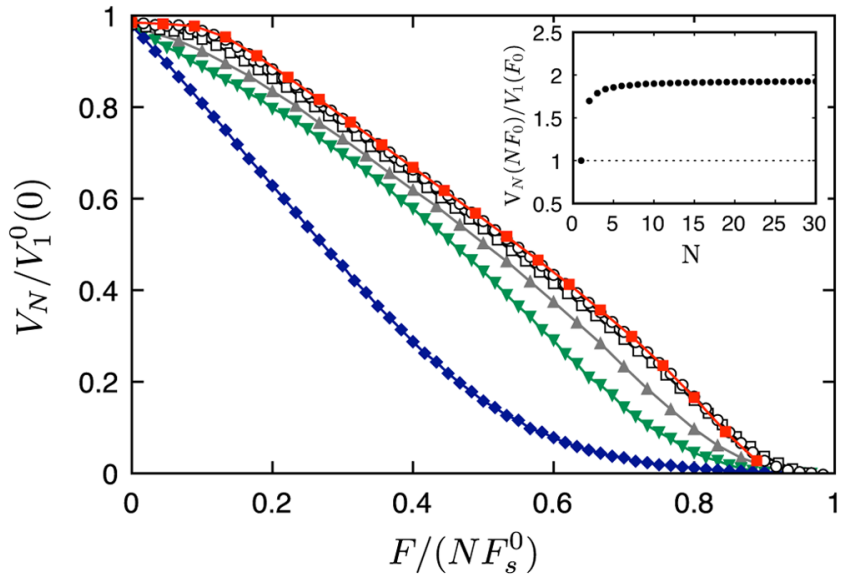

FIG. 3 (color online). Convergence with $N$ of velocity vs force per motor, for $\alpha=0.15$ and $\beta=14.1$. Diamond, $N=1$; inverted triangle, $N=2$; triangle, $N=3$; empty square, $N=5$; empty circle, $N=10$; solid square, $N=15$. Inset, $F_{0}=\frac{1}{3} F_{s}^{0}$. 
one of the two underlying pictures is appropriate for a given type of motor is an open question.

Enhanced cooperativity originates in the elementary process in which a motor in the $U_{2}$ state is pushed to the next site by a second motor sliding down the $U_{1}$ state. This provides a deterministic (not noise-driven) mechanism for the advance of the leading motor, which is potentially dominant for any ratchet model whenever noise is too weak for the leading motor to overcome the applied force, but sufficiently strong to make the unloaded motor to advance. In our fully asymmetric model, the effect is most pronounced for $D \ll 1$. For a less asymmetric ratchet with a finite distance $a$ between the minimum and maximum of $U_{1}$, the effect is also dramatic but is maximal for $\sqrt{D \tau} \sim a$, when noise is as weak as possible without suppressing the ratchet effect on the unloaded motor [20]. This mechanism competes statistically with the other crossed-state combination (the leading motor at $U_{1}$ and the trailing one at $U_{2}$ ), which is unfavorable with respect to MF. The statistical dominance of the former combination can be proven in some limits [20].

Weak attraction.-If a weak (nonbinding) attraction is added to the hard-core, a new scenario emerges. The crossed-state combination, with the first motor in $U_{1}$ and the second in $U_{2}$, may now contribute favorably, since the leading motor, as it slides down, can pull on the trailing one. This push-and-pull feedback loop further enhances cooperativity. The effect will be significant whenever the time the second motor is effectively trapped close to the first is comparable to characteristic internal times of the motor. This condition sets a balance between noise strength and attraction. The above mechanism is reminiscent of the one described for rigid or strong coupling of motors [21,22], and invoked to explain the increased velocity of motor clusters in experiments on strongly coupled KIF1A motors [18]. Note, however, that cohesion of the motor cluster is now purely dynamic, caused by the asymmetric loading of the external force $F$, but not from the motor interactions, which are unable by themselves to hold the cluster together. This has remarkable consequences since now, increasing $F$ increases the cohesion of the cluster making the cooperativity more effective, and approaching the limit of rigid coupling, for which the cluster would be faster than the single motor [18]. Consequently, the motor cluster may speed up when increasing the opposing load (see Fig. 4). This counterintuitive behavior is a genuine cooperative effect, and should be distinguished from inherent nonlinearities of the single-motor problem, which could lead in principle to similar behaviors [2,23]. Upon further increasing the load, the cluster velocity must eventually decrease, resulting in nonmonotonic velocity-force curves [24]. This behavior has strong implications for the dynamic self-organization of motor clusters when a reservoir of motors is available behind the cluster. In fact, when $F$ is increased beyond the value for which $V_{2}(F)<V_{1}(0)$, a third motor will be naturally recruited and the cluster

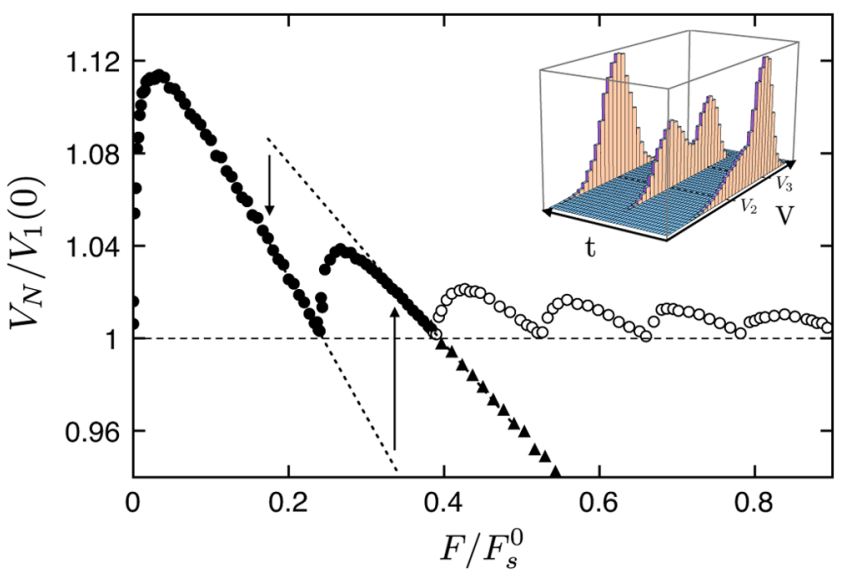

FIG. 4 (color online). Velocity-force curve for the case of attractive forces. Solid circles and triangles, $N=3$; circles (empty and solid), $N=7$. Arrows indicate hysteretic transitions from the metastable extensions for $N_{c}=2$ and $N_{c}=3$. The inset shows the transient bimodality for the decay of the 3-motor metastable cluster to the 2-motor (downward arrow). In all cases $\alpha=0.15, \beta=14.1$ and $W(\xi)=4 \epsilon\left(\left(\frac{\sigma}{\xi}\right)^{12}-\left(\frac{\sigma}{\xi}\right)^{6}\right)$ with $\sigma=0.2$ and $\epsilon=1 / 4$. Units are such that $v=3, \lambda=1, \ell=1$.

velocity will start increasing again. Consistently, for $N$ motors, the curve $V_{N}(F)$ bounces back $N-2$ times at $V_{1}(0)$ before decreasing below that value, each one implying the recruitment of an additional motor by the cluster. Figure 4 shows a genuinely self-organized collective behavior that has no counterpart within MF or for rigidly coupled motors. In the neighborhood of forces $F_{i}$ where $V_{N}\left(F_{i}\right)=V_{1}(0)$ one observes hysteresis, since the capture or release of one motor takes a finite time. The velocity distribution then exhibits transient bimodality (see Fig. 4).

Discussion.-We have identified two scenarios of motor cooperation for nonattractive and attractive interactions. In both scenarios the performance of motors is generically increased with respect to the MF additivity, the effects becoming dramatic for weak noise. The collective efficiency is also remarkably improved (see Fig. 5). In the first case only steric effects are present and the scenario is qualitatively that of MF. If a motor reservoir is available an arbitrarily large cluster will form and will be able to overcome any arbitrarily large force $F$. The total power $F V_{N}(F)$ will be typically larger than that for MF, but for fixed $F$, it will only achieve the maximal value $F V_{1}(0)$ for $N \rightarrow \infty$, that is for vanishing efficiency.

On the other hand, if a weak short-ranged attraction is included in the presence of a motor reservoir, the selforganization dynamics is fundamentally different. The velocity force is bounded from below by $V_{1}(0)$. For given $F$, the cluster dynamically selects a finite number of motors $N_{c}(F)$ that is stable and escapes from the rest. If $F$ is changed, the cluster will readjust $N_{c}$, either releasing or recruiting motors. Remarkably, in this scenario each motor in the cluster is faster than a free motor, yet it exerts a finite force. The total power delivered by the cluster is thus larger 


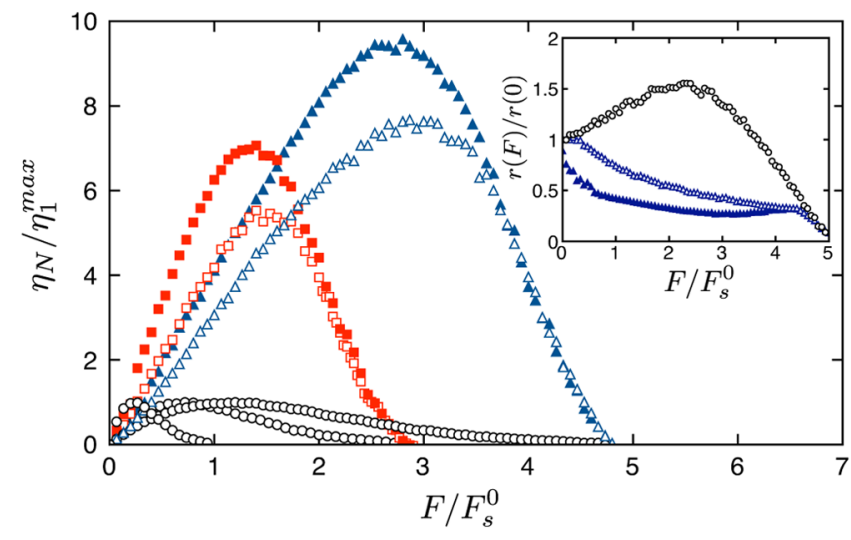

FIG. 5 (color online). Efficiency normalized to the maximum value for $N=1$. Empty circles, $N=1$ and $N=3,5$ for $\mathrm{MF}$; squares, $N=3$, triangles $N=5$ (solid symbol, attractive case, empty symbol, repulsive case). The parameters are the same of Fig. 4. In the inset, the corresponding normalized ATP consumption for 5 motors. The ATP consumption of all motors is computed, even if $F$ is such that the stable cluster $N_{c}<N$ (see text).

than the maximal value achieved for repulsive interaction with infinite $N$, that is $P \gtrsim F V_{1}(0)$. Moreover, this is now achieved with a finite number of motors, that is, with finite power per motor $P / N_{c} \simeq \Delta F V_{1}(0)$ (where $\Delta F$ is gap force for unit change in $N_{c}$ ) and with a finite efficiency. Consequently, with the possibility to recruit motors, the cluster efficiency keeps increasing monotonically with $F$. Note that this second scenario is general because it only relies on two features that can be assessed a priori, namely, that a motor pair, if rigidly bound, would be faster than a single motor, and that a proper balance between energy scales of thermal noise and attraction is fulfilled. For KIF1A, the first condition is indeed satisfied [18]. The second condition could potentially be satisfied by tuning both friction (which enters the noise strength) and interaction through modification of cargoes attached to the motors.

The reported effects apply generically to motors driven by a ratchet mechanism. Our model is minimal in terms of the number of parameters and maximizes the ratchet effect, but the simplifying assumptions are not crucial, and not even optimal for the cooperative effects discussed [20]. A quantitatively more accurate description of KIF1A, would require the introduction of additional parameters (a less asymmetric ratchet potential and a ATP-concentration-dependent transition rate). For other motors such as dimeric kinesins, similar effects could exist if a diffusive bottleneck is present in the motor cycle. For possible experiments with KIF1A, the finite processivity and force-dependent kinetics should be included. Interestingly, the present analysis provides an explicit prediction for the mean force distribution along the cluster, which shows a plateau [20]. This would justify the assump- tion of constant force share [13], implying that our predictions would directly apply to clusters smaller than the size predicted by a kinetic analysis with uniform share [13]. The interplay of cooperativity and motor kinetics is an interesting question that requires further attention.

Our general conclusion is that the collective performance of weakly coupled processive motors is optimized if the external load is unevenly distributed, in which case the cooperative action may largely outperform the simple superposition of individual motors. Whether the exploitation of the high cooperativity of monomeric kinesin may be a reason for its preference over dimeric kinesin for axonal transport in neurons, which has specific high-performance requirements, is an intriguing and interesting possibility. From a more general perspective in nonequilibrium physics, our predictions could also be observed in other nonbiological ratchet systems.

Financial support from Project FIS2006-03525 (Ministerio de Ciencia y Tecnología, Spain) is acknowledged. We are grateful to Otger Campàs for useful discussions.

[1] B. Alberts et al., The Molecular Biology of the Cell (Garland, New York, 1994).

[2] J. Howard Mechanics of Motor Proteins and the Cytoskeleton (Sinauer Press, Sunderland, MA, 2001).

[3] S. Leibler and D. A. Huse, J. Cell Biol. 121, 1357 (1993).

[4] R. D. Vale et al., Cell 59, 915 (1989).

[5] F. Jülicher and J. Prost, Phys. Rev. Lett. 75, 2618 (1995).

[6] A. Vilfan et al., Eur. Phys. J. B 3, 535 (1998).

[7] M. Badoual et al., Proc. Natl. Acad. Sci. U.S.A. 99, 6696 (2002).

[8] S. Klumpp and R. Lipowsky, Proc. Natl. Acad. Sci. U.S.A. 102, 17284 (2005).

[9] A. Roux et al., Proc. Natl. Acad. Sci. U.S.A. 99, 5394 (2002).

[10] G. Koster et al., Proc. Natl. Acad. Sci. U.S.A. 100, 15583 (2003).

[11] C. Leduc et al., Proc. Natl. Acad. Sci. U.S.A. 101, 17096 (2004).

[12] O. Campàs et al., Phys. Rev. Lett. 97, 038101 (2006).

[13] O. Campàs et al., Biophys. J. 94, 5009 (2008).

[14] P. Reimann, Phys. Rep. 361, 57 (2002).

[15] F. Jülicher et al., Rev. Mod. Phys. 69, 1269 (1997).

[16] A. Parmeggiani et al., Phys. Rev. E 60, 2127 (1999).

[17] Y. Okada and N. Hirokawa, Science 283, 1152 (1999).

[18] Y. Okada et al., Nature (London) 424, 574 (2003).

[19] K. Nishinari et al., Phys. Rev. Lett. 95, 118101 (2005).

[20] J. Brugués et al. (unpublished).

[21] A. Ajdari, J. Phys. I (France) 4, 1577 (1994).

[22] S. Klumpp et al., Phys. Rev. E 63, 031914 (2001).

[23] M.E. Fisher and A. B. Kolomeisky, Physica (Amsterdam) 274A, 241 (1999).

[24] For finite $a$, this phenomenon may be even more pronounced in the optimal regime $\sqrt{D \tau} \sim a$ [20]. 